\title{
Exotic polyquark states and their properties in QCD
}

\author{
Dmitri Melikhov ${ }^{1,2,3, \star}$ \\ ${ }^{1}$ Institute for High Energy Physics, Austrian Academy of Sciences, Nikolsdorfergasse 18, A-1050 Vienna, \\ Austria \\ ${ }^{2}$ D. V. Skobeltsyn Institute of Nuclear Physics, M. V. Lomonosov Moscow State University, 119991, Moscow, \\ Russia \\ ${ }^{3}$ Faculty of Physics, University of Vienna, Boltzmanngasse 5, A-1090 Vienna, Austria
}

\begin{abstract}
We formulate rigorous criteria for selecting diagrams to be taken into account in the analysis of potential polyquark (tetra or penta) poles in QCD. The central point of these criteria is the requirement that the Feynman diagrams for the relevant Green functions contain four- or five-quark intermediate states and the corresponding cuts. It is shown that some diagrams which "visually" seem to contain the four-quark cuts, turn out to be free of these singularities and therefore should not be taken into account when calculating the tetraquark properties. We then consider large- $N_{c}$ QCD which in many cases provide qualitatively correct picture of hadron properties and discuss in detail the tetraquark states. For the "direct" and the "recombination" four-point Green functions, which may potentially contain the tetraquark poles, we formulate large- $N_{c}$ consistency conditions which strongly restrict the behaviour of the tetraquark-to-ordinary meson transition amplitudes. In the end, these conditions allow us to obtain constraints on width of the potential tetraquark states at large $N_{c}$. We report that both flavor-exotic and cryptoexotic (i.e., flavor-nonexotic) tetraquarks, if the corresponding poles exist, have a width of order $O\left(1 / N_{c}^{2}\right)$, i.e. they should be parametrically narrower compared to the ordinary $\bar{q} q$ mesons with a width of order $O\left(1 / N_{c}\right)$. Moreover, for flavor-exotic states, the large$N_{c}$ consistency conditions require two narrow flavor-exotic states, each of these states coupling dominantly to one specific meson-meson channel.
\end{abstract}

\section{Motivation}

In the recent years, many candidates for exotic tetraquark mesons (most of the states with charmonium or bottomonium like structure, i.w. with the hidden charm or the hidden beauty) and a few candidates for pentaquark states have been reported (see [1,2]). To be more precise, the experiments have observed the resonance-like structures in the region near the two-hadron thresholds tempted to be interpreted as the new hadron poles. The precise status of these structures is still not fully understood (whether they correspond to the poles or are just complicated kinematical near-threshold effects), and the interest in the theoretical understanding of these states is incredible. The two main questions concerning the exotic states to be answered are the following: (i) do the scattering amplitudes have new poles (in the complex plane), and (ii) if the new poles exist, what is the structure of the corresponding

^e-mail: dmitri_melikhov@gmx.de 
hadrons, i.e. whether they are dominantly molecular-like states-bound states in the Van-der-Waals potential between colorless hadrons, or they are bound states of two or more colored objects in in a confined potential. Answering these questions starting from the QCD Lagrangian is an immensely difficult problem.

To provide the theoretical understanding of exotic states, one may consider QCD with a large number of colors $N_{c}$ [i.e., $\mathrm{SU}\left(N_{c}\right)$ gauge theory for large $N_{c}$ ] with a simultaneously decreasing coupling $\alpha_{s} \sim 1 / N_{c}[3,4]$ : in the sector of the ordinary hadrons, large- $N_{c}$ considerations are known to provide qualitative explanations of many phenomena of real strong interactions corresponding to $N_{c}=3$. A specific feature of the large- $N_{c}$ QCD, which simplifies the analysis of the QCD Green functions, is that at the leading order, large- $N_{c}$ QCD Green functions receive contributions only from planar diagrams (with an infinite number of gluon exchanges) and as the result have only non-interacting mesons as intermediate states; if tetraquark bound states emerge at all, they may emerge only in subleading large- $N_{c}$ diagrams [5]. For many years, this fact was believed to be an argument providing the theoretical explanation of the non-existence of exotic tetraquarks. However, it was noticed in [6], that even if the exotic tetraquark bound states appear in the subleading diagrams, the crucial question is the width of these objects: if narrow, they might still be well observed in nature. The conclusion in [6] obtained on the basis of the large- $N_{c}$ counting, that if tetraquark states exist, they may be as narrow as the ordinary mesons; the latter are known to a width $\sim 1 / N_{c}$. The question of the expected width of the exotic states (if such poles indeed exist) issue has been further addressed in [7], discussing the dependence of the width of tetraquark mesons on their flavor structure. Finally, [8] reported for the cryptoexotic tetraquarks an even smaller width of order $N_{c}^{-3}$.

A detailed analysis of the exotic states in large $N_{c}$ was performed by our group in [9]. The main content of the present talk is based on these results.

\section{Diagram selection criteria}

In order to draw a conclusion about the width of a potential tetraquark pole, one has to understand which QCD diagrams (in the general case, not only at large- $N_{c}$ ) may lead to the appearance of this pole and thus should be included in the analysis.

The crucial property of such diagrams is in fact obvious: since we are going to study bound state containing four quarks, the appropriate QCD diagrams should contain four-quark intermediate states and the corresponding cuts in the variable $s$ if we expect to observe a tetraquark pole in $s$ [10]. An unambiguous way to establish the presence (or the absence) of such a four-particle $s$-cut is to apply the Landau equations [11] which allow one to find the location of all singularities of the Feynman diagrams. A detailed application of the Landau equations shows that some of the diagrams which visually seem to contain the four-quark intermediate state and which thus have been attributed to the tetraquark pole in previous analyses, in fact do not contain the necessary four-particle cut and should be therefore excluded from the analysis of the tetraquark properties. This observation has crucial consequences for the properties of the potential tetraquark poles at large- $N_{c}$ : According to our findings [9], the tetraquark width at large $N_{c}$ does not depend on its flavor structure: both flavor-exotic and flavor-nonexotic tetraquarks have the same width of order $1 / N_{c}^{2}$.

Let us study the four-point Green functions of colorless bilinear quark currents (i.e. interpolating currents for ordinary mesons) of various flavor content.

The general four-point function depends on six kinematical variables: the four momenta squared of the external currents, $p_{1}^{2}, p_{2}^{2}, p_{1}^{\prime 2}, p_{2}^{\prime 2}, p=p_{1}+p_{2}=p_{1}^{\prime}+p_{2}^{\prime}$, and the two Mandelstam variables $s=p^{2}$ and $t=\left(p_{1}-p_{1}^{\prime}\right)^{2}$. A bound state in the $s$-channel corresponds to a pole in the variable $s$ in the four-point function. 
Now, the diagrams which potentially may contribute to the tetraquark pole at $s=M_{T}^{2}$, should satisfy the following two criteria:

1. The diagram should have a nontrivial (i.e., non-polynomial) dependence on the variable $s$.

2. The diagram should have four-quark intermediate states in the $s$-channel and the corresponding $s$-cuts starting at $s=\left(m_{1}+m_{2}+m_{3}+m_{4}\right)^{2}$, where $m_{i}$ are the masses of the quarks which are suspected to form the tetraquark bound state. The presence or absence of this cut should be established by solving the Landau equations for the corresponding diagram.

Obviously, not all diagrams in the perturbative expansion of Green functions satisfy the above criteria. It is therefore convenient to decompose these diagrams into two sets: diagrams of the first set either do not depend on $s$ or have no four-quark cut in the $s$-channel and thus cannot be related to the potential tetraquark poles; diagrams of the second set satisfy both above criteria and thus contribute to the potential tetraquark pole.

Having at hand the above criteria, we study separately two cases: tetraquarks of an exotic flavor content, i.e., built up of quarks of four different flavors, $\bar{q}_{1} q_{2} \bar{q}_{3} q_{4}$, and cryptoexotic tetraquarks, i.e those of the flavor content $\bar{q}_{1} q_{2} \bar{q}_{2} q_{3}$, carrying the same flavor as ordinary mesons. The necessity to treat flavor-exotic and cryptoexotic cases separately arises from the different topologies of the appropriate QCD diagrams in these two cases.

\section{Flavour-exotic tetraquarks}

Let us consider a bilinear quark current $j_{i j}=\bar{q}_{i} \hat{O} q_{j}$ producing a meson $M_{i j}$ of flavor content $\bar{q}_{i} q_{j}$ from the vacuum, $\left\langle 0\left|j_{i j}\right| M_{i j}\right\rangle=f_{M_{i j}}$. Here, $\hat{O}$ is a combination of Dirac matrices corresponding to the meson spin and parity. We omit all Lorentz structures as they are not important for our analysis. Important for us is that at large $N_{c}$, the meson decay constants $f_{M}$ scale as $f_{M} \sim \sqrt{N_{c}}$.

In the case of four-point functions of bilinear currents involving quarks of four different flavors, denoted by $1,2,3,4$, there are two types of Green functions: the "direct" functions $\Gamma_{\mathrm{I}}^{(\mathrm{dir})}=\left\langle j_{12}^{\dagger} j_{34}^{\dagger} j_{12} j_{34}\right\rangle$ and $\Gamma_{\mathrm{II}}^{(\mathrm{dir})}=\left\langle j_{14}^{\dagger} j_{32}^{\dagger} j_{14} j_{32}\right\rangle$, and the "recombination" functions $\Gamma^{(\mathrm{rec})}=\left\langle j_{12}^{\dagger} j_{34}^{\dagger} j_{14} j_{32}\right\rangle$ and $\Gamma^{(\mathrm{rec}) \dagger}$. It turns out that the analysis of the four-quark singularities of the "direct" Green functions is rather straightforward, whereas the analysis of the "recombination" Green functions has subtleties.

\subsection{Direct channel}

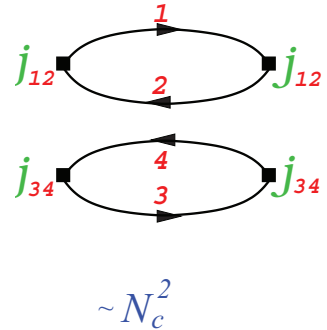

(a)

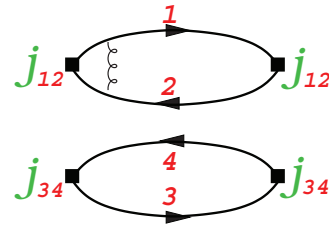

$\sim N_{c}^{3} \alpha_{s}$

(b)

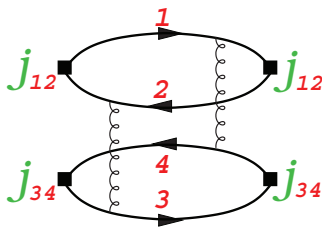

$\sim N_{c}^{2} \alpha_{s}^{2}$

(c)

Figure 1. Perturbative expansion of the "direct" Green functions $\Gamma_{I}^{(\mathrm{dir})}$. 
Figure 1 shows the perturbative expansion of the direct correlator $\Gamma_{I}^{(\mathrm{dir})}$. Similar diagrams defined by evident flavor rearrangements describe the correlator $\Gamma_{\mathrm{II}}^{(\mathrm{dir})}$. Obviously, not all these diagrams satisfy our above criteria for diagrams that potentially contain a tetraquark pole. For instance, the diagrams in Fig. 1(a,b) do not depend on $s$. The leading large- $N_{c}$ diagram which depends on $s$ and also has a four-quark $s$-cut is given by Fig. 1(c). The diagrams of this type are therefore the leading large- $N_{c}$ diagrams of interest to us.

\subsection{Recombination channel}

The analysis of the recombination channel is more involved: among the diagrams in Fig. 2, the first two diagrams $(\mathrm{a}, \mathrm{b})$ do depend on $s$; however, in spite of their appearance, they have no four-quark $s$-cut (and in fact no four-quark cuts at all).

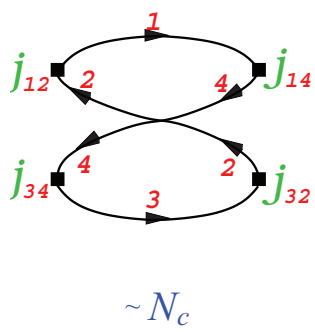

(a)

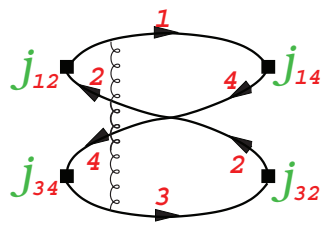

$\sim N_{c}^{2} \alpha_{s}$

(b)

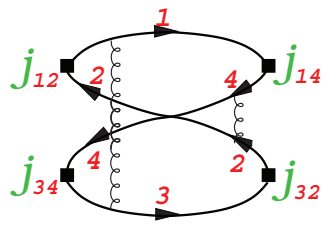

$\sim N_{c} \alpha_{s}^{2}$

(c)

Figure 2. Diagrams for the recombination Green function $\Gamma^{(\mathrm{rec})}$.

The easiest way to see this is to redraw these diagrams as the usual box diagram with gluon exchanges, see Fig. 3: The $s$-cut of the diagrams on the 1.h.s. corresponds to the $u$-cut of the box diagrams on the r.h.s. For the r.h.s. diagrams (a) and (b), the Landau equations show the absence even of the two-particle $u$-cuts; the diagrams (a) and (b) have no four-particle singularities at all. Let us emphasize that planar gluon exchanges in the diagrams on the r.h.s. of (a) and (b) do not change this property. In order to develop a four-quark cut, one needs a non-planar gluon exchange: a non-planar diagram in the r.h.s. of 3 (c) develops a four-quark $u$-cut when the crossed propagators go on-shell. Respectively, this corresponds to the four-particle $s$-cut of the 1.h.s. diagram in Fig. 3 (c). Finally, the $N_{c}$-leading diagram exhibiting the four-quark $s$-cut is the nonplanar diagram in Fig. 3(c).

Its four-quark cut with the threshold at $s=\left(m_{1}+m_{2}+m_{3}+m_{4}\right)^{2}$ may be verified directly by solving the Landau equations.

Based on the established $N_{c}$-leading diagrams possessing four-particle intermediate states, we find the following $N_{c}$-leading behaviour of the Green functions $\Gamma_{T}$ potentially involving a pole corresponding to some tetraquark $T$ :

$$
\begin{aligned}
\Gamma_{\mathrm{I}, T}^{(\mathrm{dir})} & =\left\langle j_{12}^{\dagger} j_{34}^{\dagger} j_{12} j_{34}\right\rangle=O\left(N_{c}^{0}\right), \\
\Gamma_{\mathrm{II}, T}^{(\mathrm{dir})} & =\left\langle j_{14}^{\dagger} j_{32}^{\dagger} j_{14} j_{32}\right\rangle=O\left(N_{c}^{0}\right), \\
\Gamma_{T}^{(\mathrm{rec})} & =\left\langle j_{12}^{\dagger} j_{34}^{\dagger} j_{14} j_{32}\right\rangle=O\left(N_{c}^{-1}\right) .
\end{aligned}
$$

These diagrams potentially contain the tetraquark pole, although the actual existence of this pole is still a conjecture. Now, let us assume that narrow resonances (i.e., resonances with widths vanishing 


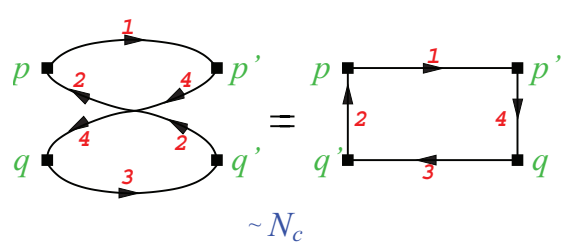

(a)

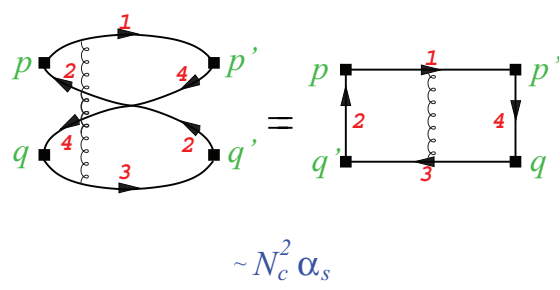

(b)

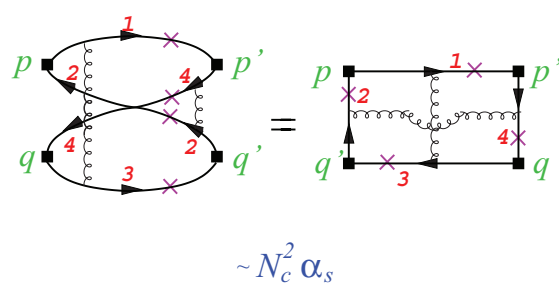

(c)

Figure 3. Diagrams for the recombination Green function $\Gamma^{(\mathrm{rec})}$ displayed as box diagrams.

for large $N_{c}$ ) show up at the lowest possible $1 / N_{c}$ order and that the resonance mass $M_{T}$ remains finite at large $N_{c}$. The fact that the "direct" and the "recombination" Green functions of flavour-exotic currents have different large- $N_{c}$ behaviours, leads to the conclusion that a single pole cannot saturate the leading behaviour of both the direct and the recombination Green functions and we need at least two exotic poles, denoted by $T_{A}$ and $T_{B}$ : one state, $T_{A}$, couples stronger to the $M_{12} M_{34}$ channel, while another state, $T_{B}$ couples stronger to the $M_{14} M_{32}$ channel.

Truncating the poles corresponding to the external mesons and retaining explicitly only the tetraquark poles, we get

$$
\begin{gathered}
\Gamma_{\mathrm{I}, T}^{(\mathrm{dir})}=O\left(N_{c}^{0}\right)=f_{M}^{4}\left(\frac{\left|A\left(M_{12} M_{34} \rightarrow T_{A}\right)\right|^{2}}{p^{2}-M_{T_{A}}^{2}}+\frac{\left|A\left(M_{12} M_{34} \rightarrow T_{B}\right)\right|^{2}}{p^{2}-M_{T_{B}}^{2}}\right)+\cdots, \\
\Gamma_{\mathrm{II}, T}^{(\mathrm{dir})}=O\left(N_{c}^{0}\right)=f_{M}^{4}\left(\frac{\left|A\left(M_{14} M_{32} \rightarrow T_{A}\right)\right|^{2}}{p^{2}-M_{T_{A}}^{2}}+\frac{\left|A\left(M_{14} M_{32} \rightarrow T_{B}\right)\right|^{2}}{p^{2}-M_{T_{B}}^{2}}\right)+\cdots, \\
\Gamma_{T}^{\text {(rec) }=}=O\left(N_{c}^{-1}\right)=f_{M}^{4}\left(\frac{A\left(M_{12} M_{34} \rightarrow T_{A}\right) A\left(T_{A} \rightarrow M_{14} M_{32}\right)}{p^{2}-M_{T_{A}}^{2}}\right. \\
\left.+\frac{A\left(M_{12} M_{34} \rightarrow T_{B}\right) A\left(T_{B} \rightarrow M_{14} M_{32}\right)}{p^{2}-M_{T_{B}}^{2}}\right)+\cdots .
\end{gathered}
$$


At this point we have to take into account the scaling relation $f_{M} \sim \sqrt{N_{c}}$. Now, since we are interested in the properties of the potential tetraquark poles with finite mass at large $N_{c}$, the equations (2) have the following solution:

$$
\begin{array}{ll}
A\left(T_{A} \rightarrow M_{12} M_{34}\right)=O\left(N_{c}^{-1}\right), & A\left(T_{A} \rightarrow M_{14} M_{32}\right)=O\left(N_{c}^{-2}\right), \\
A\left(T_{B} \rightarrow M_{12} M_{34}\right)=O\left(N_{c}^{-2}\right), & A\left(T_{B} \rightarrow M_{14} M_{32}\right)=O\left(N_{c}^{-1}\right) .
\end{array}
$$

The widths $\Gamma\left(T_{A, B}\right)$ of the states $T_{A}$ and $T_{B}$ are determined by the dominant channel. We therefore conclude that both states have parametrically the same widths, $\Gamma\left(T_{A, B}\right)=O\left(N_{c}^{-2}\right)$.

So far we have ignored the mixing between $T_{A}$ and $T_{B}$. Introducing their mixing parameter $g_{A B}$, we get additional contributions to the above Green functions. Most restrictive for $g_{A B}$ is the recombination function, for which mixing provides the additional contribution

$$
\Gamma_{T}^{(\mathrm{rec})}=O\left(N_{c}^{-1}\right)=f_{M}^{4}\left(\frac{A\left(M_{12} M_{34} \rightarrow T_{A}\right)}{p^{2}-M_{T_{A}}^{2}} g_{A B} \frac{A\left(T_{B} \rightarrow M_{14} M_{32}\right)}{p^{2}-M_{T_{B}}^{2}}\right)+\cdots .
$$

Equations (3) and (4) restrict the behaviour of the mixing parameter in the following way:

$$
g_{A B} \leq O\left(N_{c}^{-1}\right) .
$$

Thus, the two flavor-exotic tetraquarks of the same flavor content do not mix at large $N_{c}$.

\section{Cryptoexotic tetraquarks}

Let us now discuss tetraquarks with nonexotic flavor content, i.e., having the same flavor as the ordinary mesons. All confirmed tetraquark candidates observed experimentally belong to this class of states. The analysis proceeds along the same line as for the exotic states. There is however an important new ingredient compared to flavour-exotic states: diagrams of new topologies compared to the flavor-exotic case emerge.

\subsection{Direct channel}

For the direct Green functions $\Gamma_{(\mathrm{I}, \mathrm{II}), T}^{(\mathrm{dir})}$ the new diagrams are shown in Fig. 4. Essentail for us is that the new diagrams do not change the leading large- $N_{c}$ behaviour compared to the diagrams of the same topology as in the flavor-exotic case.

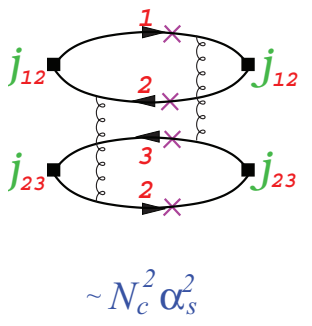

(a)

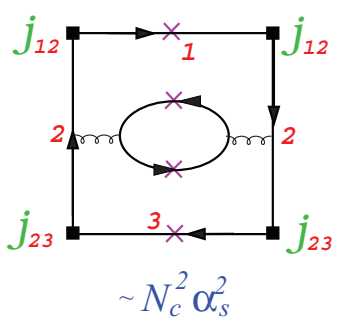

(b)

Figure 4. Diagrams contributing to $\Gamma_{\mathrm{I}, T}^{(\mathrm{dir})}$. Crossed propagators denote on-shell particles contributing to the four-quark cut. 


\subsection{Recombination channel}

For the recombination functions, however, the situation changes qualitatively: the new diagram, Fig. 5(b), dominates at large $N_{c}$ and thus modifies the leading large- $N_{c}$ behaviour of $\Gamma_{T}^{(\mathrm{rec})}$. We thus find

$$
\begin{aligned}
& \Gamma_{\mathrm{I}, T}^{(\mathrm{dir})}=\left\langle j_{12}^{\dagger} j_{23}^{\dagger} j_{12} j_{23}\right\rangle=O\left(N_{c}^{0}\right), \\
& \Gamma_{\mathrm{II}, T}^{(\mathrm{dir})}=\left\langle j_{13}^{\dagger} j_{22}^{\dagger} j_{13} j_{22}\right\rangle=O\left(N_{c}^{0}\right), \\
& \Gamma_{T}^{(\mathrm{rec})}=\left\langle j_{12}^{\dagger} j_{23}^{\dagger} j_{13} j_{22}\right\rangle=O\left(N_{c}^{0}\right) .
\end{aligned}
$$

In contrast to the flavor-exotic case, now both the direct and the recombination Green functions have the same leading behaviour at large $N_{c}$. As a consequence, one exotic state $T$ is already sufficient in order to satisfy the expected large- $N_{c}$ behaviour of both Green functions. The couplings of this state to the meson-meson channels are

$$
\begin{aligned}
& A\left(T \rightarrow M_{12} M_{23}\right)=O\left(N_{c}^{-1}\right), \\
& A\left(T \rightarrow M_{13} M_{22}\right)=O\left(N_{c}^{-1}\right) .
\end{aligned}
$$

The exotic state $T$ is then expected to have coupling of the order to both possible decay channel, i.e. no preferrable decay channel is seen. The width of this single cryptoexotic state is of order $\Gamma(T)=O\left(N_{c}^{-2}\right)$.

If all its quantum numbers allow, $T$ can mix with the ordinary meson $M_{13}$. The restriction on the mixing parameter $g_{T M_{13}}$ may be obtained, e.g., from the direct amplitude

$$
\Gamma_{\mathrm{I}, T}^{(\mathrm{dir})}=O\left(N_{c}^{0}\right)=f_{M}^{4}\left(\frac{A\left(M_{12} M_{23} \rightarrow T\right)}{p^{2}-M_{T}^{2}} g_{T M_{13}} \frac{A\left(M_{13} \rightarrow M_{12} M_{23}\right)}{p^{2}-M_{M_{13}}^{2}}\right)+\cdots
$$

Taking into account that $A\left(M_{13} \rightarrow M_{12} M_{23}\right) \sim 1 / \sqrt{N_{c}}$ [3, 4], we obtain for the mixing parameter

$$
g_{T M_{13}} \leq O\left(1 / \sqrt{N_{c}}\right)
$$

Thus, the cryptoexotic tetraquark and the ordinary meson with the same flavour do not mix at large $N_{c}$.

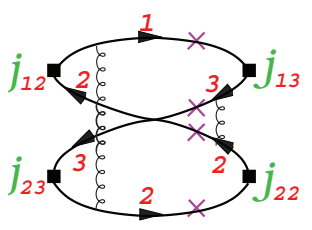

$\sim N_{c} \alpha_{s}^{2}$

(a)

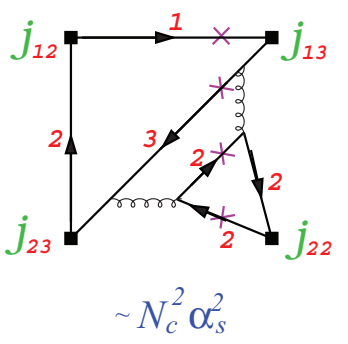

(b)

Figure 5. Diagrams contributing to the recombination Green function $\Gamma_{T}^{(\mathrm{rec})}$. 


\section{Conclusions and outlook}

We have formulated a set of physically obvious and rigorous criteria for selecting Feynman diagram appropriate for the analysis of possible tetraquark (i.e. a bound state containing four quarks, independently of the structure of this bound state): namely, if one is going to study properties of the tetraquark, only those diagrams, which contain four-quark intermediate states and the corresponding four-quark cuts in the appropriate variable should be taken into account. Diagrams which do not contain the four-quark intermediate states cannot contribute to the appearance of the tetraquark pole and should therefore be neglected.

In particular, when one discusses four-point functions of the bilinear quark currents, describing the scattering of the ordinary $\bar{q} q$-mesons, one should consider only those contributions to the four-point Green functions which have a four-quark cut in the $s$-channel. The straightforward and rigorous way to establish these four-quark singularities is provided by the Landau equations. These simple criteria are quite general and are applicable in QCD for any $N_{c}$.

Now, these general criteria for the diagram selection, combined with the large- $N_{c}$ limit, provide essential constraints on the properties of the possible exotic tetraquark mesons. Namely, using these criteria and requiring that the narrow poles contribute to the appropriate parts of the Green functions at the leading large- $N_{c}$ order, we reported the following results:

1. One has important consistency conditions which arise from the large- $N_{c}$ behaviour of the direct and the recombination four-point Green functions. These consistency conditions restrict the number of exotic states necessary to saturate the leading large- $N_{c}$ behaviour of the four-point Green functions.

2. For the flavor-exotic four-point Green functions (all four quarks of different flavors $\left.\bar{q}_{1}, \bar{q}_{3}, q_{2}, q_{4}\right)$, their large- $N_{c}$ behaviour is not compatible with the existence of only one flavorexotic tetraquark bound state; the consistency condition but requires two narrow states $T_{A}$ and $T_{B}$ with widths $\Gamma\left(T_{A}, T_{B}\right)=O\left(N_{c}^{-2}\right)$. Each of these tetraquarks dominantly couples to one meson-meson channel; its coupling to the other meson-meson channel is suppressed: $A\left(T_{A} \rightarrow M_{12} M_{34}\right)=O\left(N_{c}^{-1}\right), A\left(T_{A} \rightarrow M_{14} M_{32}\right)=O\left(N_{c}^{-2}\right), A\left(T_{B} \rightarrow M_{14} M_{32}\right)=O\left(N_{c}^{-1}\right)$, $A\left(T_{B} \rightarrow M_{12} M_{34}\right)=O\left(N_{c}^{-2}\right)$. The parameter describing the mixing between the two tetraquarks vanishes for large $N_{c}$ at least like $1 / N_{c}$.

3. The large- $N_{c}$ behaviour of four-point Green functions of nonexotic flavor content $\left(\bar{q}_{1}, \bar{q}_{2}, q_{2}, q_{3}\right)$ is compatible with the existence of a single narrow cryptoexotic tetraquark, $T$, with width $\Gamma(T)=O\left(N_{c}^{-2}\right)$. This tetraquark couples parametrically equally to both two-meson channels: $A\left(T \rightarrow M_{12} M_{23}\right)=O\left(N_{c}^{-1}\right), A\left(T \rightarrow M_{13} M_{22}\right)=O\left(N_{c}^{-1}\right)$. If quantum numbers allow, the cryptoexotic tetraquark $T=\bar{q}_{1} q_{3} \bar{q}_{2} q_{2}$ mixes with the ordinary meson $M_{13}=\bar{q}_{1} q_{3}$. The corresponding mixing parameter vanishes like $1 / \sqrt{N_{c}}$, i.e., slower than that of the flavor-exotic tetraquarks.

4. The constraints on the width of the possible narrow exotic states at large $N_{c}$ are general and universal, i.e. they do not depend on the structure of the tetraquark pole. In particular, the constraints should be satisifed by both molecular and confined tetraquarks.

5. We discussed the consequences of the large- $N_{c}$ behaviour of the four-point Green functions for the narrow exotic states with the mass staying finite at large $N_{c}$. However, the constraints on the Green functions are quite general and cannot be violated by any exotic tetraquarks, including those with the masses which rise with $N_{c}$. In this case, however, one should take into account that the number of the open channels with many ordinary mesons in the final states also rises 
with $N_{c}$. Therefore, obtaining constraints on the width of the exotic states with the mass rising with $N_{c}$ needs a detailed treatment.

We would like to mention that, in principle, there is a possibility that narrow tetraquarks do exist but appear only in $N_{c}$-subleading diagrams with four-quark intermediate states, while they do not contribute to the $N_{c}$-leading topologies. This possibility seems rather unnatural to us: if such pole exists at all, there should be some special reason, not evident to us, why it does not appear in the set of the appropriate $N_{c}$-leading diagrams. Nevertheless, also in the $N_{c}$-subleading topologies one observes a difference in the large- $N_{c}$ behaviour of the direct and the recombination diagrams of the flavor-exotic case: for any $N_{c}$-subleading topology, the direct diagrams are $N_{c}$-even, whereas the recombination diagrams are $N_{c}$-odd. Accordingly, if the latter scenario is realized in nature, one still encounters the necessity of two flavor-exotic poles, although with parametrically smaller widths at large $N_{c}$.

Acknowledgements. I have pleasure to thank W. Lucha and H. Sazdjian for a fruitful collaboration on the subject of this talk. Valuable discussions with V. Anisovich, T. Cohen, M. Knecht, B. Moussallam, O. Nachtmann, and B. Stech are gratefully acknowledged. The work was supported by the Austrian Science Fund (FWF) under project P29028.

\section{References}

[1] S. L. Olsen, Front. Phys. (Beijing) 10, 101401 (2015).

[2] A. Ali, J. S. Lange, and S. Stone, arXiv:1706.00610 [hep-ph].

[3] G. 't Hooft, Nucl. Phys. B72, 461 (1974).

[4] E. Witten, Nucl. Phys. B160, 57 (1979).

[5] S. Coleman, Aspects of symmetry (Cambridge University Press, Cambridge, England, 1985), Chapter 8.

[6] S. Weinberg, Phys. Rev. Lett. 110, 261601 (2013).

[7] M. Knecht and S. Peris, Phys. Rev. D 88, 036016 (2013).

[8] L. Maiani, A. D. Polosa, and V. Riquer, JHEP 1606, 160 (2016).

[9] W. Lucha, D. Melikhov, and H. Sazdjian, Phys. Rev. D 96, 014022 (2017).

[10] T. D. Cohen and R. F. Lebed, Phys. Rev. D 90, 016001 (2014).

[11] L. D. Landau, Nucl. Phys. 13, 181 (1959). 